\section{Lymphozytäres Infiltrat als Prognosefaktor}

Tumorzellen entgehen nicht immer dem Schutzmechanismus des körpereigenen Immunsystems. Wie Art und Ausmaß der lymphozytären Infiltration eines lokal begrenzten, malignen Melanoms das weitere Tumorgeschehen beeinflussen, wurde in einer aktuellen australischen Studie untersucht.

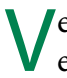
erlässliche Prognosefaktoren sind eine wichtige Voraussetzung für das weitere diagnostische Prozedere, für die Therapieentscheidung und für die $\mathrm{Zu}-$ teilung von Patienten in Risikogruppen. Neben Dicke des Primärtumors, Mitoserate, möglicher Ulzeration, anatomischer Lokalisation oder bestimmten $\mathrm{Pa}$ tientencharakteristika gilt der SentinelLymphknotenstatus (SLN-Status) als ein wichtiger Prognosefaktor. In der vorliegenden Studie wurde untersucht, ob die Präsenz und die Verteilung von tumorinfiltrierenden Lymphozyten (TILs) im malignen Gewebe als unabhängige Prädiktoren für den SLN-Status und das Gesamtüberleben zu werten sind.

Die Autoren griffen auf die Datenbank des Melanoma Institute Australia zurück und identifizierten 1.865 Patienten im Alter von median 60 Jahren, denen zwischen 2001 und 2006 ein primäres kutanes Melanom mit einer Tumordicke von $0,75 \mathrm{~mm}$ oder mehr chirurgisch entfernt worden war. Untersucht wurde der Einfluss klinischer und pathologischer Faktoren auf den SLN-Status, das rezidivfreie Überleben und das melanomspezifische Überleben. Den TIL-Grad bestimmten sie anhand eines VierPunkte-Schemas entsprechend der Dichte (leicht, moderat, ausgeprägt) und Verteilung (fokal, multifokal, diffus) des lymphozytären Infiltrats:

_ Grad 0: nicht nachweisbar,

- Grad 1: TILs leicht oder moderat fo$\mathrm{kal} / \mathrm{mild}$ multifokal,

_ Grad 2: TILs ausgeprägt fokal/moderat oder ausgeprägt multifokal/leicht diffus,

_ Grad 3: TILs moderat oder ausgeprägt diffus.

Bei der Mehrzahl der Patienten konnten entweder keine (TIL Grad 0: 35,4\%) oder nur in geringem Ausmaß (TIL Grad 1:
$45 \%)$ tumorinfiltrierende Lymphozyten nachgewiesen werden. Nur rund $3 \%$ der Patienten entsprachen dem TIL-Grad 3. Weitere Ergebnisse: Je geringer die Tumordicke, Mitoserate und Eindringtiefe des Tumors, desto höher war der TILGrad. Zudem korrelierte der SLN-Status invers mit dem TIL-Grad.

Prädiktoren für einen positiven Sentinel-Lymphknotennachweis waren ein jüngeres Lebensalter, niedrigere TILGrade, Ulzeration, höhere Tumordicke, Satellitose und erhöhte Mitoseraten. Die Raten für das melanomspezifische und rezidivfreie Überleben nach fünf Jahren lagen bei 83 bzw. $76 \%$, für die Patientengruppe mit einem TIL-Grad 3 hingegen bei $100 \%$.

Fazit: Der TIL-Grad als Indikator für die Dichte und Verteilung lymphozytärer Infiltrate im Tumorgewebe ist ein unabhängiger Prädiktor für das Gesamtüberleben und den SLN-Status bei Melanom-Patienten.

Wolfgang Zimmermann

Farhad A et al. Tumor-infiltrating lymphocyte grade is an independent predictor of sentinel lymph node status and survival in patients with cutaneous melanoma. J Clin Oncol. 2012; 30(21):2678-83.

\title{
Neues Target im BRAF-Signaltransduktionsweg
}

Bei etwa der Hälfte aller Patienten mit einem fortgeschrittenen malignen Melanom finden sich aktivierende Mutationen in der Serin/Threonin-Proteinkinase BRAF. Hemmer der MEK-Kinase könnten das Therapiespektrum bei diesem problematischen Patientenkreis erweitern.

Clektive BRAF-Inhibitoren markier$\checkmark$ ten einen ersten Fortschritt in der Therapie von Melanompatienten und verbesserten Gesamt- und progressionsfreies Überleben im Vergleich zu chemotherapeutischen Ansätzen, aber meist mit zeitlich begrenztem Response. Als alternative Targets im fehlgeleiteten MAP-Kinase-Signaltransduktionsweg bieten sich die MEK-Proteine 1 und 2 an, die durch das mutierte BRAF aktiviert werden und das Tumorwachstum beeinflussen.

In Phase-I- und -II-Studien führte der orale selektive MEK1/MEK2-Inhibitor Trametinib zu Tumorregression und Stabilisierung der Erkrankung bei fortge- schrittenen Melanomen mit BRAF-Mutationen.

In einer randomisierten, kontrollierten, offenen Phase-III-Studie erhielten 322 Melanom-Patienten mit den genannten molekularen Charakteristika entweder den MEK-Inhibitor (2 mg oral) einmal täglich oder Dacarbazin (1.000 $\left.\mathrm{mg} / \mathrm{m}^{2}\right)$ oder Paclitaxel $\left(175 \mathrm{mg} / \mathrm{m}^{2}\right)$ i.v. alle drei Wochen. Für Patienten mit progredienter Erkrankung unter Chemotherapie war ein Crossover zu Trametinib vorgesehen. Primärer Endpunkt war das progressionsfreie Überleben.

Das mediane Gesamtüberleben in der Trametinib-Gruppe betrug 4,8 Monate gegenüber nur 1,5 Monaten in der Chemotherapie-Gruppe. Nach sechs Monaten lag die Gesamtüberlebensrate nach Trametinib bei $81 \%$, in der Vergleichsgruppe trotz Crossover bei $67 \%$. Zu den häufigsten Nebenwirkungen des MEKInhibitors zählten Exanthem, Diarrhö und periphere Ödeme, jedoch keine sekundären kutanen Neoplasmen.

Fazit: Die Therapie mit dem oralen selektiven MEK-Inhibitor Trametinib kann progressionsfreies und Gesamtüberleben von Patienten mit metastasiertem malignem Melanom mit BRAF-Mutation (V600E oder V600K) verbessern. Eventuelle Kombinationen mit BRAFInhibitoren bedürfen weiterführender Studien.

Wolfgang Zimmermann

Flaherty KT et al. Improved survival with MEK inhibition in BRAF-mutated melanoma. N Engl J Med. 2012;367(2):107-14. 